\title{
Environmental impoverishment and aging alter object recognition, spatial learning, and dentate gyrus astrocytes
}

Daniel G. Diniz, ${ }^{1}$ César A. R. Foro, ${ }^{1}$ Carla M. D. Rego, ${ }^{1}$ David A. Gloria, ${ }^{2}$ Fabio R. R. de Oliveira, ${ }^{1}$ Juliana M. P. Paes, ${ }^{1}$ Aline A. de Sousa, ${ }^{1}$ Tatyana P. Tokuhashi, ${ }^{1}$ Lucas S. Trindade, ${ }^{3}$ Maíra C. P. Turiel, ${ }^{1}$ Erick G. R. Vasconcelos, ${ }^{2}$ João B. Torres, ${ }^{1}$ Colm Cunnigham, ${ }^{4}$ Victor H. Perry, ${ }^{5}$ Pedro F. da Costa Vasconcelos ${ }^{6}$ and Cristovam W. P. Diniz ${ }^{1}$ ${ }^{1}$ Universidade Federal do Pará-UFPA, Instituto de Ciências Biológicas, Laboratório de Investigações em Neurodegeneração e Infecção, Hospital Universitário João de Barros Barreto, Brazil

${ }^{2}$ Universidade Federal do Pará-UFPA, Curso de Licenciatura em Ciências Biológicas, Instituto de Estudos Costeiros, Campus de Bragança, Brazil

${ }^{3}$ Universidade do Estado do Pará-UEPA, Centro de Ciências Biológicas e da Saúde CCBS, Belém, Pará, Brazil

${ }^{4}$ Department of Biochemistry, Trinity College Institute of Neuroscience, Trinity College, Dublin 2, Ireland

${ }^{5}$ Southampton Neuroscience Group, School of Biological Sciences, University of Southampton, Southampton SO16 7PX, UK

${ }^{6}$ Instituto Evandro Chagas, IEC, Departamento de Arbovirologia e Febres Hemorrágicas, Ananindeua, Pará, Brazil

Keywords: albino Swiss mice, enriched environment, impoverished environment, memory

\begin{abstract}
Environmental and age-related effects on learning and memory were analysed and compared with changes observed in astrocyte laminar distribution in the dentate gyrus. Aged (20 months) and young (6 months) adult female albino Swiss mice were housed from weaning either in impoverished conditions or in enriched conditions, and tested for episodic-like and water maze spatial memories. After these behavioral tests, brain hippocampal sections were immunolabeled for glial fibrillary acid protein to identify astrocytes. The effects of environmental enrichment on episodic-like memory were not dependent on age, and may protect water maze spatial learning and memory from declines induced by aging or impoverished environment. In the dentate gyrus, the number of astrocytes increased with both aging and enriched environment in the molecular layer, increased only with aging in the polymorphic layer, and was unchanged in the granular layer. We suggest that long-term experience-induced glial plasticity by enriched environment may represent at least part of the circuitry groundwork for improvements in behavioral performance in the aged mice brain.
\end{abstract}

\section{Introduction}

The environmental conditions under which animals are reared affect subsequent cognitive performance (Kempermann et al., 1997; Duffy et al., 2001; Teather et al., 2002). An enriched environment has been defined as that which offers social interactions with conspecifics and stimulation of exploratory and motor behavior with periodic changes in the variety of toys, ladders, tunnels, ropes, bridges and running wheels for voluntary physical exercise. In contrast, an impoverished environment offers standard cages with reduced sensorial, motor and cognitive stimulation (van Praag et al., 2000). Compared with those reared in impoverished environments, rodents reared in enriched environments exhibit increases in brain size and weight, the number of neurons in the dentate gyrus, the number and area of synapses, the number and density of dendritic branches, and the production of neurotrophic factors (Rosenzweig \& Bennett, 1996; Kolb \& Whishaw, 1998; van Praag et al., 2000; Rampon \& Tsien, 2000).

Correspondence: Dr C. Wanderley Picanço Diniz, as above.

E-mail: cwpdiniz@gmail.com

Received 28 January 2010, revised 8 April 2010, accepted 26 April 2010
Studies of environmental effects on brain plasticity have focused on altered neuronal morphology; however, substantial morphological changes have also been shown to occur in glial cells (Sirevaag \& Greenough, 1991; Komitova et al., 2002), and the first report to describe glial multiplication associated with enriched environment was published in 1964 using autoradiography (Altman \& Das, 1964).

Integrated studies involving aging, environment and glial changes have recently become popular because astrocytes are implicated in a number of local hippocampal regulatory processes that affect learning and memory (Junjaud et al., 2006; Magistretti, 2006; Mothet et al., 2006; Todd et al., 2006; Perea \& Araque, 2007). Indeed, in the murine hippocampus, astrocytes are by far the most numerous of glial cells, and several studies have revealed age-related increases in astrocytes (Pilegaard \& Ladefoged, 1996; Long et al., 1998b; Mouton et al., 2002). In the rat hippocampus, it was shown that astrocytes were smaller in size and number in aged rats raised in enriched environments compared with age-matched controls (Soffie et al., 1999). On the other hand, astrocytes in the CA1 region of adult mice raised in an enriched environment presented similar numbers, but longer and more ramified branches, than the controls housed in standard cages (Viola 
et al., 2009). It has been previously suggested that astrocytes exhibit transient responses to the individual's stress history rather than longterm adaptive responses to differential effects of rearing in complex or laboratory cage environments (Sirevaag \& Greenough, 1991). These and other data (Chadashvili \& Peterson, 2006; Billard \& Rouaud, 2007) demonstrate that we lack understanding regarding associations between non-neuronal plasticity and the effects of environment and aging (Markham \& Greenough, 2004). Many questions remain unresolved, including the quantitative relationships between changes in morphology and behavior and between changes in astrocyte number and environment and aging-related factors. Indeed, no studies have reported simultaneous (unbiased) measurements of the number of dentate gyrus astrocytes and assessments of behavior in murine models of aging raised in different environments.

In the present report, we assessed the integrated memories of young adult (6 months) and aged (20 months) mice raised in either enriched or impoverished environments, and we investigated whether affected memories were correlated with changes in the number of astrocytes in the dentate gyrus.

\section{Materials and methods}

\section{Subjects and experimental groups}

Seventy-one albino Swiss female young adult (6 months) and aged (20 months) mice were housed from weaning in enriched conditions (EC, $n=27$ ) or impoverished conditions (IC, $n=29$ ). These formed four experimental groups: enriched environment, young adults (EY, $n=12$ ); impoverished environment, young adults (IY, $n=13$ ); enriched environment, aged adults (EA, $n=15$ ); and impoverished environment, aged adults (IA, $n=16$ ). EC comprised two-level wire cages $(100 \times 50 \times 100 \mathrm{~cm})$, equipped with ropes, rod bridges, tunnels, running wheels and toys. Toys were made of different forms of plastic, wood and metal of different colors, and were changed periodically. Each EC cage housed 12-15 young and aged mice. Water and food were delivered to the top and bottom levels, respectively. This obliged mice to move from one compartment to another for drinking and eating.

IC comprised plastic cages $(32 \times 39 \times 100 \mathrm{~cm})$ without equipment or toys. Each IC cage housed 12-13 young and aged mice. All mice had free access to water and food. In addition, 12-h dark and light cycles were maintained. Behavioral tests were given during the light cycle.

\section{Behavioral procedures}

We used the episodic-like memory test to assess integrative memories (Dere et al., 2005a), and a long-term water maze test to assess learning and spatial memory (Morris, 1984). All young adult (EY and IY) and aged (EA and IA) mice were used in both tests.

The apparatus for the episodic-like memory test consisted of an open box $(30 \times 30 \times 40 \mathrm{~cm})$ made of painted white wood. The floor was painted with black lines to form nine squares $(10 \times 10 \mathrm{~cm})$, and the luminance at the center of the cage floor was $2.4 \mathrm{~cd} / \mathrm{m}^{2}$. Detailed protocols and the rationale for test choices were discussed elsewhere (Dere et al., 2005a,b). In brief, behavioral assays were performed after 12 days: 7 days to become accustomed to handling, 3 days for open field habituation, 2 days for object habituation, and then 1 day of testing.

\section{Handling}

Each day, mice were placed in the center of the arena for $1 \mathrm{~min}$ and then removed to their cages.

\section{Open field habituation}

Each day, mice were placed in the arena, free of objects, for $5 \mathrm{~min}$ to explore the open field.

\section{Object habituation}

Each day, mice were exposed to two identical objects placed at the corners of the arena for $5 \mathrm{~min}$, three times, with $50 \mathrm{~min}$ in between. These objects were not used on the test days.

\section{Testing}

The episodic-like memory test was administered once for each mouse. In order to minimize the influence of natural preferences for particular objects or materials, we chose objects of the same material, with different geometries that could be easily discriminated, and similar access for interaction (Dere et al., 2005a). All objects were made of plastic with different shapes, heights and colors. Before each mouse entered the arena, the box and objects were cleaned with $75 \%$ ethanol to minimize distinguishing olfactory cues.

\section{Episodic-like memory test}

A diagram of the episodic-like memory test is shown in Fig. 1. This test consisted of three trials: two 5-min sample trials and one 5-min test trial, with 50-min intervals between trials. In the first sample trial, four identical objects were presented. In the second sample trial, four different identical objects were presented. In the test trial, two objects from sample trial 1 ('old' objects) and two objects from sample trial 2 ('recent' objects) were presented. In addition, one object from the first sample trial was shifted to a new location ('displaced' object), but the objects from the second sample trial remained in the same places ('stationary' objects). It was expected that mice would spend more time with objects from the first sample trial (old objects) than those from the second sample trial (recent objects), and would show a preference for the 'old, displaced' object, a second preference for the 'old, stationary' object, and finally equal preferences for the 'recent stationary' objects. In the episodic-like memory test the exploration of an object was assumed when a mouse approached an object, the head was directed towards it, and the head was placed within $0-3 \mathrm{~cm}$ from the object. This definition required that each object be fixed to the apparatus floor, thus we chose heavy objects for interaction. The performance was defined as the percentage of time spent exploring one object. To account for individual variability in exploratory activity, the time spent with each object was normalized by the total exploration time for each individual.

Detailed statistical procedures for episodic-like memory tests protocol were described elsewhere (Dere et al., 2005a). In brief, for the episodic-like memory test, the basic measure obtained from videoimages was the time a mouse spent during the test trial in explorations of the 'old', 'recent', 'displaced' and 'stationary' objects. The performance was the time of exploration for each object, expressed as a proportion (percentage) of the total time of exploration. Possible significant differences were also detected with the two-tailed $t$-test for dependent groups (Dix \& Aggleton, 1999).

\section{Water maze test}

A different set of young (EY, $n=12$; IY, $n=13$ ) and aged (EA, $n=15$; IA, $n=11$ ) adult mice groups were trained in the water maze adapted for mouse dimensions. The circular pool and platform were 94 and $14 \mathrm{~cm}$ in diameter, respectively, and the platform was $1 \mathrm{~cm}$ below the water surface. To occlude the platform, the pool was filled with dark-blue water $\left(22 \pm 2^{\circ} \mathrm{C}\right)$ colored with a non-toxic dye. In each trial, 


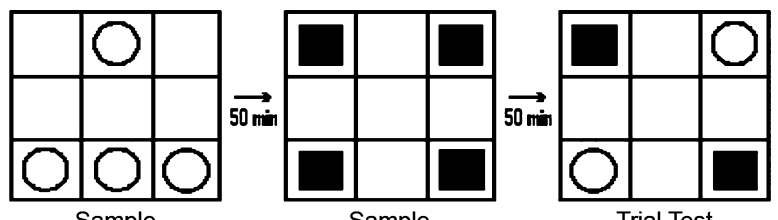

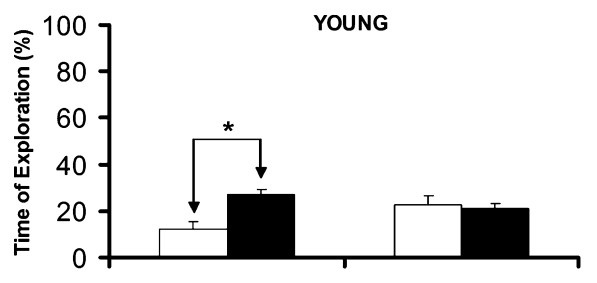

EC

ISTATIONARY IDISPLACED

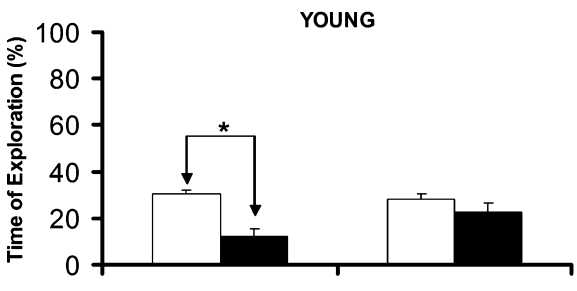

EC

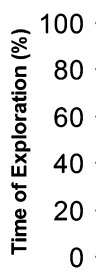

$\left.\begin{array}{r}100 \\ 80 \\ 60 \\ 40 \\ 20 \\ 0\end{array}\right]$
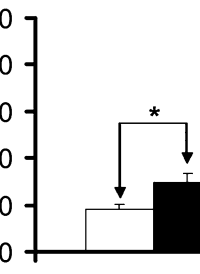

EC

口STATIONARY - DISPLACED

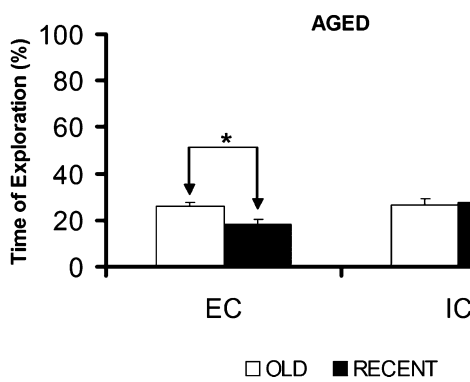

FIG. 1. Top: diagram of the experimental designs for integrated tests (episodic-like memory). In the first sample (left panel), mice explored four identical objects (open circles) placed in the configuration shown. In the second sample (middle panel), mice explored four different identical objects (filled squares) placed in a different configuration. In the test trial (right panel), mice explored two objects from trial 1 ('old' objects; open circles), one placed in its former position (stationary) and one in a new position (displaced); and two objects from trial 2 ('recent' objects; filled squares), both placed in their former positions (stationary; modified from Dere et al., 2005a). Bottom: results from episodic-like memory tests. Values on the $y$-axes represent the time of exploration for each object as a percentage of the total time of exploration. Labels on the $x$-axes indicate experimental groups. Bars indicate average values \pm SEM for the indicated groups. Filled and open bars represent different types of objects (displaced vs. stationary; old vs. recent). ${ }^{*}$ Two-tailed $t$-test; $P<0.05$. EC, enriched conditions; IC, impoverished conditions.

the subjects were allowed 1.5 min to find the hidden platform; trials were separated by intervals of $90 \mathrm{~s}$; the task was considered complete when animals found and remained on the platform for $5 \mathrm{~s}$. The first day of water maze training was dedicated to adapting the animal to the aquatic labyrinth. In the remaining 7 days, animals were tested once per day in four trials. The learning rate ' $C$ ' for the water maze was assessed by measuring four trials of escape latency and comparing results measured on the 1 st and 5 th test days. The ratio was denoted as $C$, the contrast index, calculated with the following equation:

$$
C=\frac{(L 1-L 5)}{(L 1+L 5)},
$$

where $L 1$ and $L 5$ are the escape latencies or total distance traveled to find the platform measured on the 1 st and 5 th test days, respectively. The contrast index was used to normalize the learning curve to each individual's performance, thus accounting for the variation in performances between individuals (Torres et al., 2006). Raw data were also included to illustrate individual performances in the water maze tests and compare with contrast values.

We recorded escape latency, distance traveled, average swimming speed and trajectories for each mouse. All groups were compared using one-way ANOVA, Bonferroni a priori test or two-way ANOVA followed by Bonferroni post hoc tests, with differences between groups accepted as significant at a $95 \%$ confidence level $(P<0.05)$.
All tests were recorded with a webcam, and images were analysed with a computer program to score the time spent interacting with objects and performance in the water maze (ANYMAZE tracking system, Stöelting). Computer analysis was performed offline.

\section{Perfusion and histological procedures}

At the end of the water behavioral tests, all subjects were weighed and killed with an overdose of ketamine $(100 \mathrm{mg} / \mathrm{kg})$ and xylazine (10 mg/kg; Konig Laboratories). They were then perfused transcardially with heparinized saline for $10 \mathrm{~min}$, followed by an aldehyde fixative (4\% paraformaldehyde in $0.1 \mathrm{M}$ phosphate buffer, $\mathrm{pH}$ 7.2-7.4) for $30 \mathrm{~min}$. All chemicals were purchased from Sigma (São Paulo, Brazil). After perfusion and craniotomy, the brains were removed and cut on a vibratome at a $70 \mu \mathrm{m}$ thickness. One out of each five sections was used to detect glial fibrillary acid protein (GFAP) by free-floating immunohistochemistry. Free-floating sections were rinsed once in $0.1 \mathrm{M}$ phosphate buffer, transferred to $0.2 \mathrm{M}$ boric acid $\mathrm{pH} 9.0$, heated to $65-70^{\circ} \mathrm{C}$ for $1 \mathrm{~h}$, and then washed three times, 5 min each, in PBST (5\%). The sections were incubated under constant shaking in a $1 \%$ hydrogen peroxide solution in methanol for $10 \mathrm{~min}$, then rinsed twice, 2 min each, in $0.1 \mathrm{M}$ PBS. The sections were then blocked with immunoglobulin for $1 \mathrm{~h}$, according to the instructions for the Mouseon-Mouse Immunodetection kit (M.O.M. kit, Vector Laboratories, 
USA). Blocking was followed by three washes, 2 min each, in PBS. Sections were incubated in a working solution of protein concentrate for $5 \mathrm{~min}$, then incubated with monoclonal mouse anti-GFAP primary antibody (MAB360, CHEMICON Int, USA), diluted $1: 800$ in protein concentrate solution (M.O.M. kit), at $4^{\circ} \mathrm{C}$ for 3 days with continuous, gentle agitation. Next, the sections were washed three times, 2 min each, in PBS and incubated for $20 \mathrm{~h}$ with the biotinylated horse anti-mouse secondary antibody (M.O.M. kit), diluted $1: 100$ in PBS. After three washes, $2 \mathrm{~min}$ each, in PBS, sections were transferred to an avidin-biotin-peroxidase complex solution $(\mathrm{ABC}$, Vector Laboratories, USA; $1: 200$ ) for $1.5 \mathrm{~h}$, washed three times, $2 \mathrm{~min}$ each, in $0.1 \mathrm{M}$ PBS, and processed with the glucose oxidasediaminobenzidine-nickel method and peroxidase histochemistry (Shu et al., 1988).

The reaction was interrupted after fine astrocytic branches were detected under the microscope. Sections were rinsed four times, $5 \mathrm{~min}$ each, in $0.1 \mathrm{M}$ PBS, mounted on gelatinized slides, dehydrated in alcohol and xylene, and coverslipped with Enthelan (Merck).

\section{Photomicrographic documentation and processing}

Digital photomicrographs were taken with a digital camera (Microfire, Optronics, CA, USA) coupled to a Nikon microscope (Optiphot-2, NY, USA). Digital photomicrographs were processed with Adobe Photoshop 7.0.1 C.S.2 software (San Jose, CA, USA) for scaling and adjusting the levels of brightness and contrast applied to the whole image.

The selected micrographs display representative sections from each experimental group in which the astrocyte number in each region of interest was closest to the mean value for that region.

\section{Microscopy and optical fractionator}

Details of the optical fractionator methodology, experimental parameters and results for each subject are described in online supplementary material (Tables S1-S6).

In brief, we delineated at all levels in the histological sections the region and layers of dentate gyrus, digitizing directly from sections using low-power $3.2 \times$ objective on a Optiphot-2 microscope (Nikon, Japan) equipped with a motorized stage (MAC200, Ludl Electronic Products, Hawthorne, NY, USA). This system was coupled to a computer running Stereoinvestigator software (MicroBrightField, Williston, VT, USA) used to store and analyze $x, y$ and $z$ coordinates of digitized points. In order to detect and count unambiguously the objects of interest in the dissector probe, low-power objective was replaced by a $60 \times$ oil immersion planapochromatic objective (NIKON, NA 1.4) to count astrocytes.

\section{Area and objects of interest.}

The border between the polymorphic layer and the CA3 region was arbitrarily defined in horizontal sections with a straight line that connected the tip of the pyramidal cell layer of the CA3 with the two tips of the granular cell layer. CA3 pyramidal and dentate gyrus granular layers appeared as darker bands that were easily distinguished from the polymorphic layer. The straight line was used to distinguish the astrocytes of the CA3 from the astrocytes of the polymorphic layer. All other layers of the dentate gyrus were conspicuously distinguished from each other, and individual astrocytes were promptly identified on the sections immunoreacted for GFAP.

\section{Results}

\section{Behavioral assays}

Episodic-like memory test

Only young adult and aged mice from the enriched environment were able to integrate object recognition in a spatio-temporal context. The subjects from impoverished environments were unable to make the appropriate distinctions (Fig. 1). In the spatial memory component of episodic-like memory, young and aged subjects housed in EC spent, respectively, more time in the displaced than stationary objects (EY, $26.96 \pm 2.37$ vs. $12.37 \pm 2.96, t_{11}=3.98, P=0.003$; EA, $29.65 \pm 3.81$ vs. $18.33 \pm 2.07, t_{13}=2.29, P=0.039$ ). In the identity and temporal memory components, young and aged subjects housed in EC spent, respectively, more time in the old than recent objects (EY, $30.33 \pm 1.96$ vs. $12.37 \pm 2.96, t_{11}=3.84, P=0.004 ; \quad \mathrm{EA}$, $26.00 \pm 1.81$ vs. $\left.18.33 \pm 2.07, t_{13}=2.55, P=0.024\right)$.

\section{Water maze spatial memory tests}

The water maze tested the impact of the environment and aging on the ability of an animal to learn and remember the position of a hidden platform. Figure 2A illustrates the results for the water maze performance in each experimental group, and the learning rate (contrast index) was based on escape latencies. The IY, EY and EA groups were able to learn and remember the position of the hidden platform, but the IA group did not learn the position of the platform. The tracking of the swimming trajectories at the 5th day in Fig. 2B revealed that an enriched environment improved the ability to devise a strategy for reaching the hidden platform in both young and aged groups. Figure $2 \mathrm{C}$ represents individual absolute values (black circles) and the average of daily distance (m) for each group (squares). Note that, related to the 1 st day, IY and EY reduced significantly the traveled distance on the 3 rd day (IY mean diff. $=2.28, P \leq 0.01$; EY mean diff. $=1.27, P \leq 0.05$ ), whereas EA and IA only in the 5 th (mean diff. $=1.49, P \leq 0.05$ ) and 7 th (mean diff. $=1.27, P \leq 0.05$ ) days, respectively.

Two-way ANOVA applied to the learning rate revealed that age $\left(F_{1,45}=14.71, \quad P=0.0004\right)$ and environment $\left(F_{1,45}=4.61\right.$, $P=0.037)$ affected the performances in the water maze, with no interaction between those variables $\left(F_{1,45}=0.12, P=0.73\right.$; Bonferroni post hoc tests, $P>0.05$ ).

\section{Regions and object of interest: stereological assessment}

The optical fractionator principles were applied to immunolabeled sections to quantify the GFAP astrocytic marker; thus, we estimated the total number of astrocytes in the molecular, granular and polymorphic layers of the dentate gyrus.

Figure 3 is a photomicrograph to illustrate objects of interest (GFAP-labeled astrocytes) of molecular, granular and polymorphic layer of dentate gyrus. Note the different morphologies of astrocytes in the different layers.

Figure 4 shows low- and medium-magnification photomicrographs of representative horizontal sections from the dentate gyrus and hippocampus of all experimental groups. Pictures are inverted to negative contrast to improve the visualization of the astrocyte distribution in the layers of interest. The dentate gyrus granular layer and pyramidal cell layer of CA1 and CA3 (boxed areas) appear as dark bands of low astrocytic density located between the molecular and the polymorphic layers of the dentate gyrus, and between the oriens and radiatum of CA1 and CA3 layers, respectively. All layers 
A

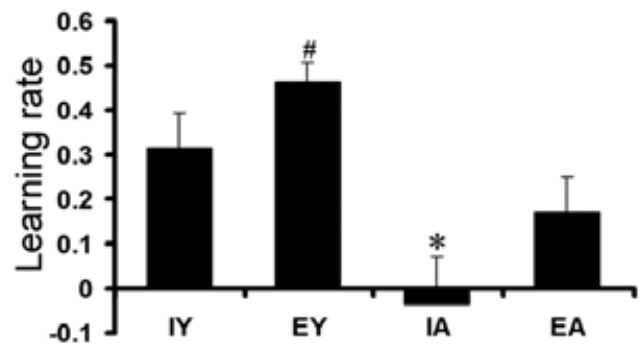

B
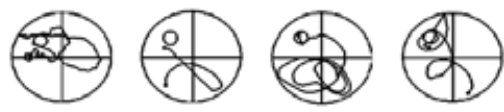

IY

EY

IA

EA

C

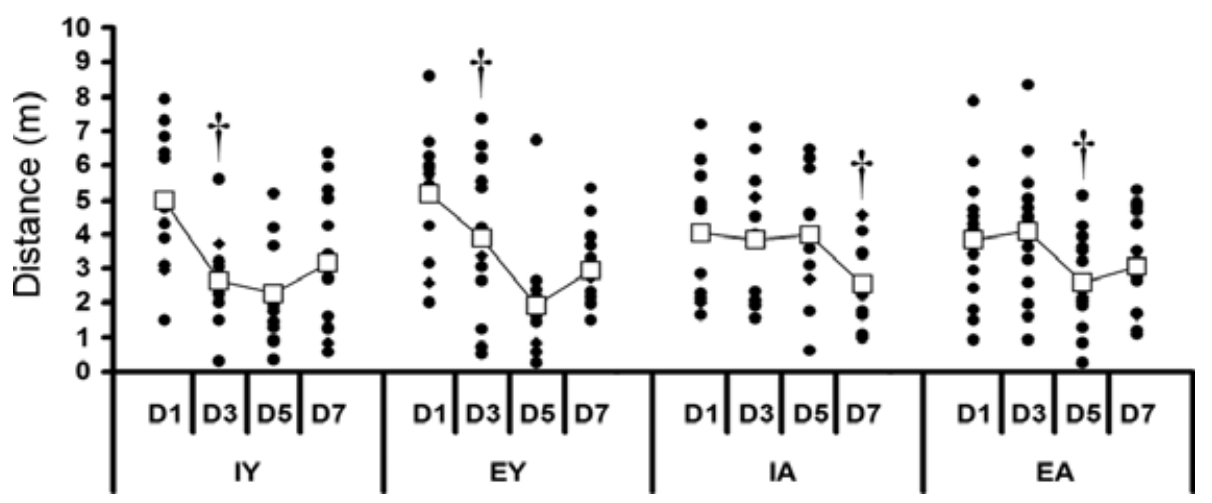

FIG. 2. Results from the water maze test. (A) The $y$-axes indicate the learning rate $[C=(L 1-L 5) /(L 1+L 5)]$ between the 1 st and 5 th test days for escape latency. Note that IA did not reduce escape latency whereas all other groups presented significant learning. Overall, EY showed a higher learning rate than all other groups. (B) Swimming trajectory tracks based on the average for each experimental group; the small circles in the upper left-hand quadrants represent the hidden platform. (C) Absolute values (black circles) of daily distance (m) traveled of each subject; white squares represent the average of the experimental group. Significant reductions of total traveled distance were observed on the 3rd, 5th and 7th days for young (EY, IY), EA and IA, respectively. * and \# indicate significant differences in comparison to all other groups (two-way ANOvA, $P<0.05$ ). ${ }^{\dagger}$ (one-way ANOVA, $P<0.05$, Bonferroni a priori test). IA, impoverished environment, aged mice; EA, enriched environment, aged mice; IY, impoverished environment, young mice; EY, enriched environment, young mice.

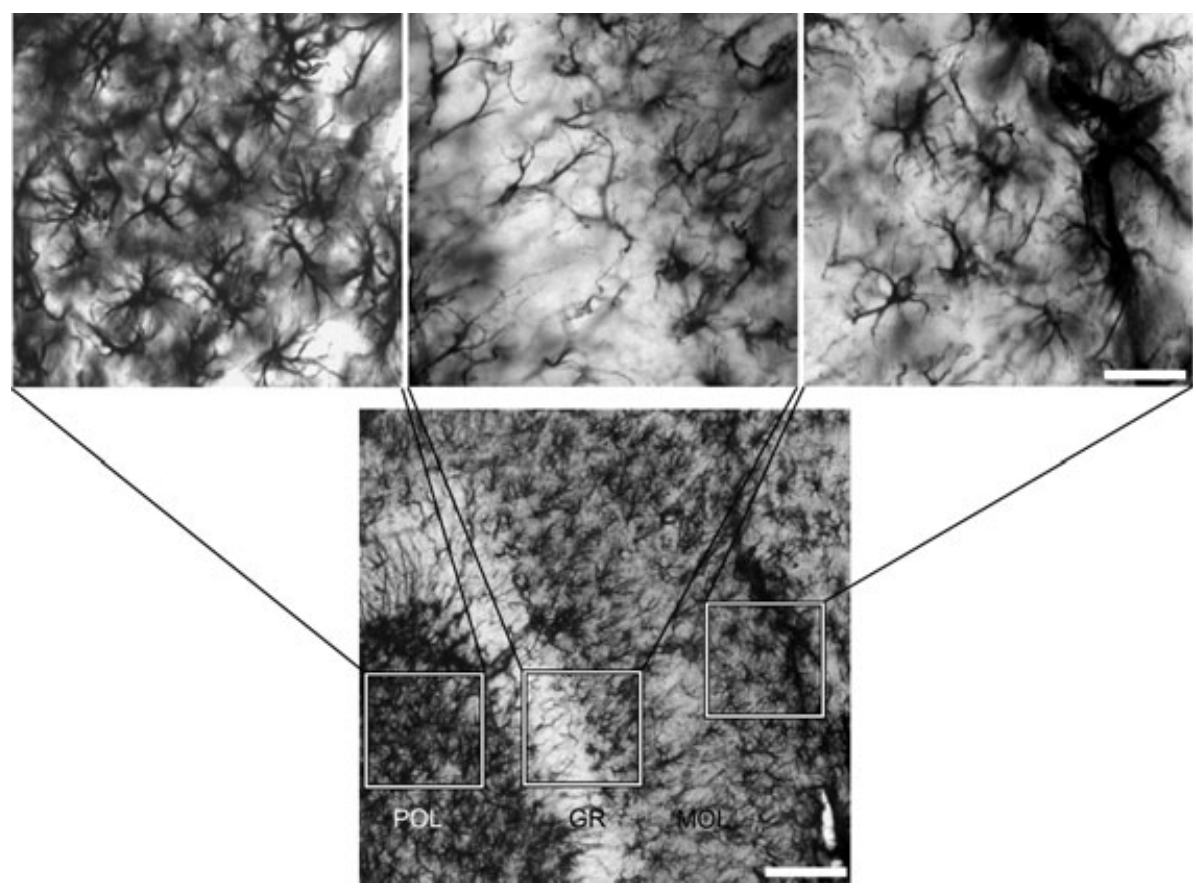

FIG. 3. Low- and high-magnification photomicrographs of a horizontal section from the hippocampal dentate gyrus immunolabeled for GFAP (astrocytic marker) to indicate the object of interest (in black). The layers of interest are labeled in the figure as Gr, granular layer; Mol, molecular layer; Pol, polymorphic layer. The boxed areas in the low-magnification panel (bottom) are shown at higher resolution in the insets (top). Note the different morphologies of astrocytes in the different layers. Scale bar: $100 \mu \mathrm{m}$ (low magnification); $50 \mu \mathrm{m}$ (high magnification). 

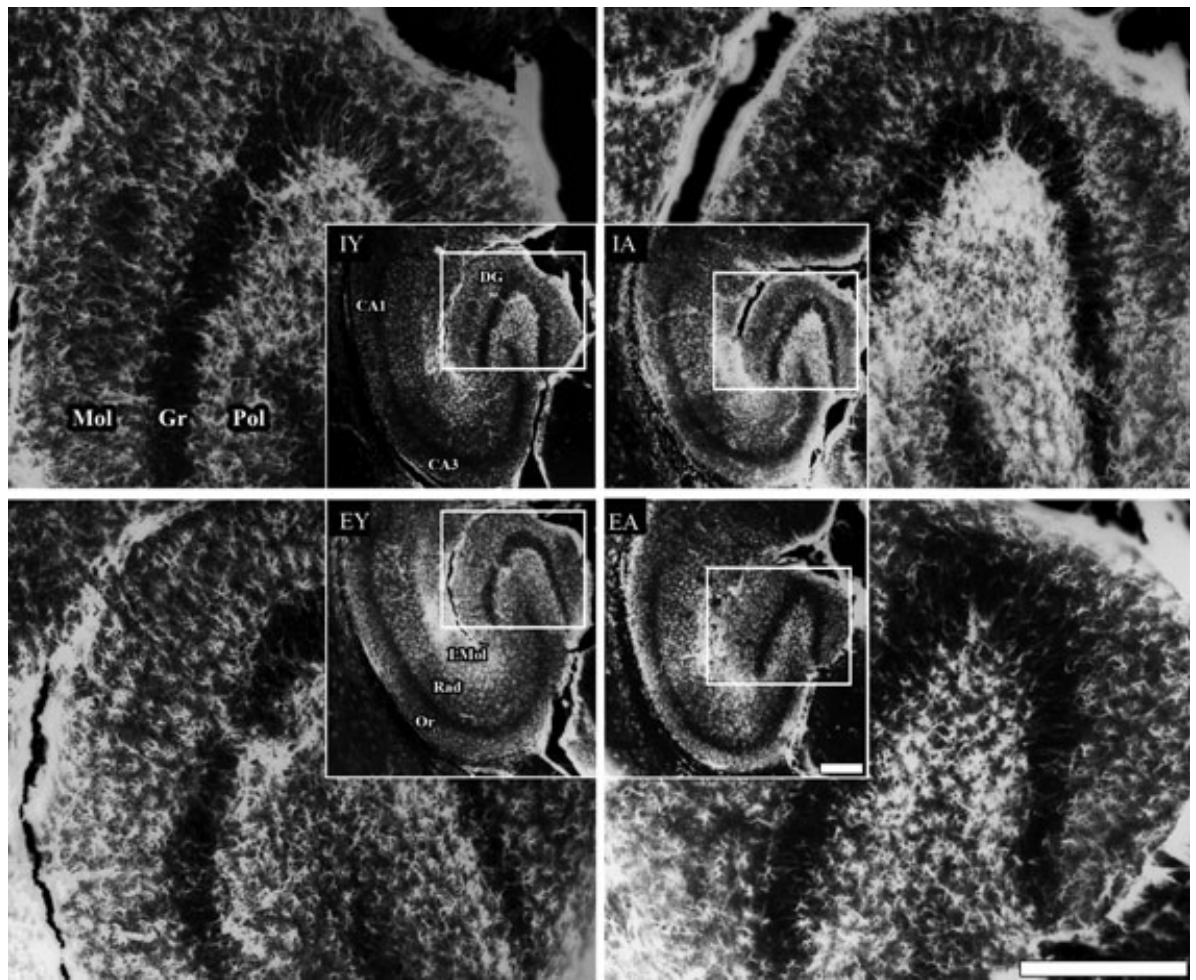

FIG. 4. Low- and medium-magnification photomicrographs from hippocampus and dentate gyrus of representative horizontal sections of each experimental group immunolabeled for GFAP. GFAP-labeled cells appear white in negative contrast. The objects of interest were the astrocytes of dentate gyrus layers indicated as Gr, granular; Mol, molecular; Pol, polymorphic. In the boxed areas the dentate gyrus (DG), CA1 and CA3 are indicated. Note that the lacunosum molecular layers of CA1 and CA3 near the hippocampal fissure show higher expression of GFAP in IA, EY and EA groups, whereas the polymorphic layers show higher expression of GFAP in the IA group. LMol, lacunosum molecular layers; Or, oriens; Rad, radiatum. IA, impoverished environment, aged mice; EA, enriched environment, aged mice; IY, impoverished environment, young mice; EY, enriched environment, young mice. Scale bars: $250 \mu \mathrm{m}$.

of the dentate gyrus, regions of interest to the stereology assessment, were easily distinguished in all counted sections of all experimental groups. Thus, we established the limits of interest between the counted layers with no ambiguity. The medium-magnification pictures illustrate typical astrocytes of different layers and give a small sample of the morphological diversity among these cells in the dentate gyrus of albino Swiss mice. Note that the lacunosum molecular layer of CA1 and CA3 near the hippocampal fissure (boxed areas) revealed the higher expression of GFAP in IA, EY and EA groups, whereas the polymorphic layer present the higher expression of GFAP in the IA group. Although to a lesser extent, EC subjects, in comparison with IC, present higher expression of GFAP in the stratum lucidum of CA3.

Supporting Tables S4-S6 in the online supplementary material contain the estimations of the total numbers of astrocytes of the molecular, granular and polymorphic layers of the dentate gyrus for each experimental group. Direct comparative analysis of those values indicated that the molecular layer was affected by both long-term environmental and aging conditions. A higher number of astrocytes was induced by an enriched environment compared with those induced with aging. Indeed, the average differences induced by environmental changes in groups EY vs. IY or EA vs. IA were 4819 and 6177 astrocytes, respectively. In contrast, the differences induced by aging in groups EA vs. EY or IA vs. IY were 4507 and 3149 astrocytes, respectively. However, in the polymorphic layer, only aging induced environmental changes (IA vs. IY), with an average of $37 \%$ more astrocytes in the aged group compared with the young group. Finally, in the granular layer, neither environmental nor aging induced significant differences among groups.
Figure 5 gives graphic representations of the total numbers of astrocytes in the molecular and polymorphic layers of the dentate gyrus; this facilitates a comparison of significant results. On average, aged mice from the enriched environment exhibited higher numbers of astrocytes in the molecular layer than young mice housed in a similar environment. Aging and enriched environment seem to promote an additive hyperplasia of astrocytes in this layer (Fig. 5A). Indeed, the estimations of the astrocytes number in the molecular layer of the dentate gyrus revealed significant differences induced by age $\left(F_{1,16}=9.24, \quad P=0.0071\right)$ and environmental $\left(F_{1,16}=12.04\right.$, $P=0.0027)$ changes, but no interactions among these variables $\left(F_{1,16}=0.45, P=0.51\right)$. In contrast, the polymorphic layer was only affected by aging with a higher number of astrocytes in aged compared with young subjects (one-way ANOVA, $F_{3,19}=3.26$, $P \leq 0.05$; Fig. 5B).

When two-way ANOVA was applied to the total number of astrocytes of the dentate gyrus, it was found that enrichment affected significantly the results $\left(F_{1,16}=6.22, P=0.024\right)$, whereas aging revealed only a tendency in the same direction $\left(F_{1,16}=4.24\right.$, $P=0.056$ ). Although a significant increase in the total number of dentate gyrus astrocytes was only affected by environment, two-tailed $t$-test revealed significant differences $\left(t_{8}=2.54, P=0.035\right)$ between the estimations of IA and IY subjects.

In all cases, the variance introduced by methodological procedures was less than $50 \%$ of the observed group variance, giving a ratio of $\mathrm{CE}^{2} / \mathrm{CV}^{2}<0.5$ (Slomianka \& West, 2005). The biological variation represented $77-99.6 \%$ of the total variation.

Figure 6 illustrates average behavioral performances in the episodic-like memory test and the number of astrocytes in the molecular 

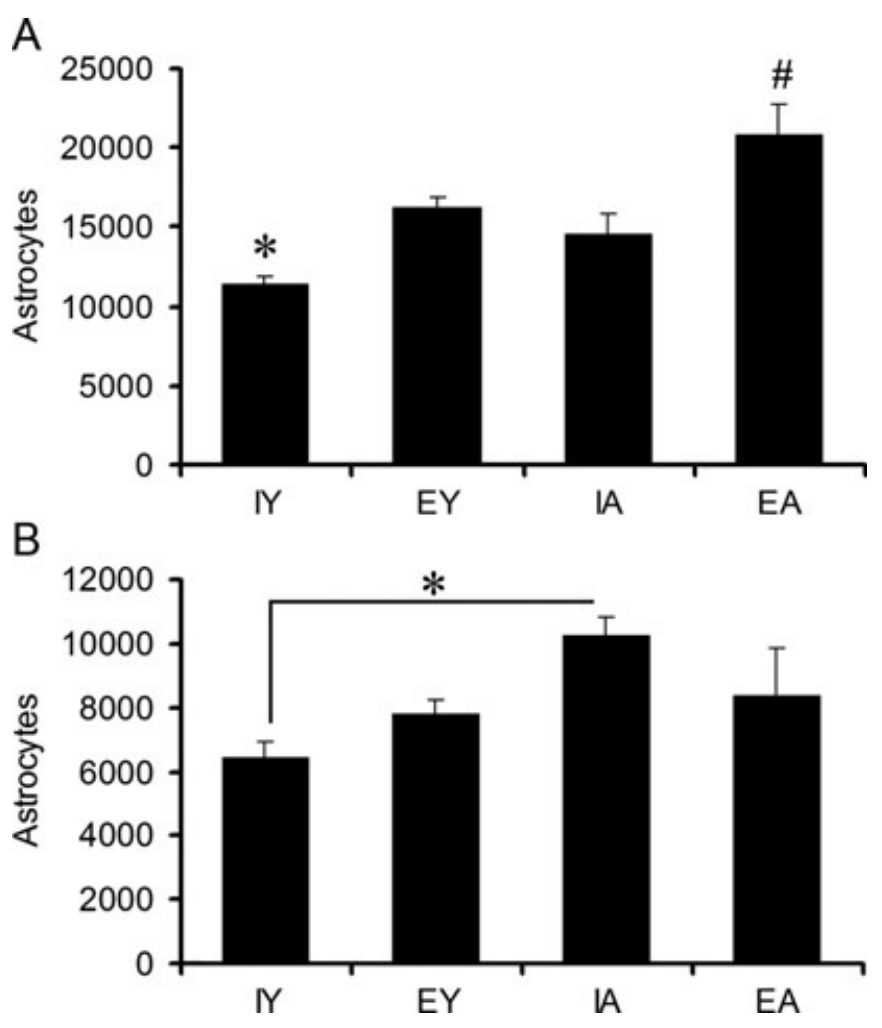

FIG. 5. Graphic representation of the average values of the estimations of astrocyte number in (A) the molecular layer and (B) the polymorphic layer of the dentate gyrus. Note the additive effects of enriched environment and aging on the number of astrocytes in the molecular layer, and the effect of aging, but not environment, on the number of astrocytes in the polymorphic layer. * and \# indicate significant differences in comparison to all other groups, two-way ANOVA, Bonferroni, $P<0.05$. * in brackets indicates significant difference between IY and IA, two-way ANOVA, Bonferroni $P<0.05$. IA, impoverished environment, aged mice; EA, enriched environment, aged mice; IY, impoverished environment, young mice; EY, enriched environment, young mice.

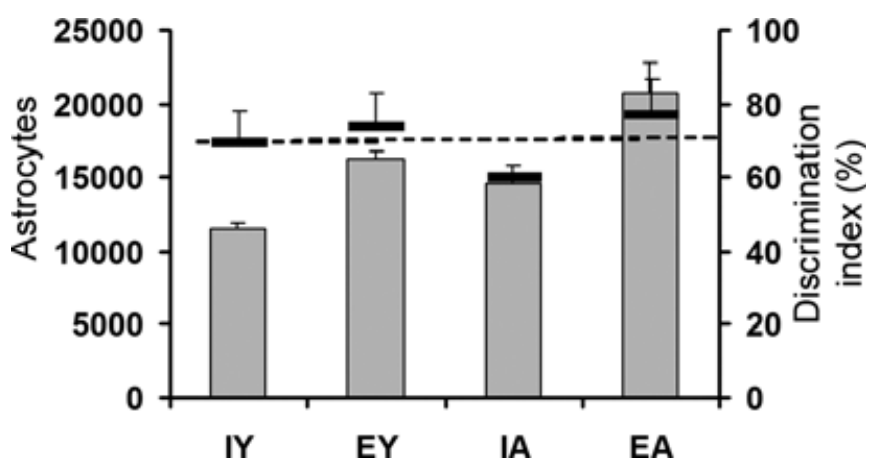

FIG. 6. Graphic representation of the average values of episodic memory-like test (right $y$-axis) and number of astrocytes in the molecular layer of the dentate gyrus (left $y$-axis) of five subjects of each experimental group. Points above $70 \%$ (dotted line) indicate the experimental group that completed the object recognition task. Note that there is no simple correlation between the number of astrocytes and behavioral performances. IA, impoverished environment, aged mice; EA, enriched environment, aged mice; IY, impoverished environment, young mice; EY, enriched environment, young mice.

layer of the dentate gyrus of the same subjects from different experimental groups. We defined as $70 \%$ the minimal acceptable difference in the time of exploration between the objects to indicate that the object recognition had occurred. The dotted line in the figure points to this level, indicating that on average IA subjects did not reach the criteria. Note that the average values of discrimination index reproduced in the selected subjects for counting, the same tendencies observed in the water maze and episodic-like memory tests. However, we did not find a simple correlation between the astrocytes numbers and episodic-like memory performances.

\section{Discussion}

The results of the present study demonstrated that a $24 \mathrm{~h} /$ day complex environmental enrichment had differential effects on the number of astrocytes in the dentate gyrus and on the performances in episodiclike and water maze memory tests among young and aged female albino Swiss mice. After 20 months, mice reared under IC exhibited cognitive dysfunctions in both tests. After 6 months, mice reared under IC also exhibited changes in the performances in episodic-like memory and water maze tests. In contrast, continuous environmental enrichment, beginning early in life (21st postnatal day), induced astrocyte hyperplasia in the molecular layer, preserved episodic-like memory and reduced the impact of aging on the Morris water maze spatial memory. Taken together the results suggested that different degrees of laminar astrocytosis were induced in the murine dentate gyrus by environmental and aging conditions. It also suggests that an enriched environment may protect against abnormal cognitive development and cognitive decline due to aging. Because aged subjects raised in EC had improved memories compared with those raised in IC, we speculate that the astrocytosis detected in these conditions may arise from different astrocytic phenotypes.

Cognitive performances of adult $\mathrm{C} 57 \mathrm{Bl} / 6$ mice were previously assessed with an integrative episodic-like memory test (Dere et al., 2006). Adult, but not aged, albino Swiss mice were previously investigated for spatial memory and learning (Koopmans et al., 2003; Rao et al., 2005; Prediger et al., 2007), but not for episodic-like memory. Thus, the present report is the first evidence in young adult albino Swiss mice that integrative episodic-like memories and, to a lesser extent, water maze learning and memory (Morris, 1984) are especially susceptible to deterioration after living in an impoverished environment for 6 months or more. In addition to the behavioral studies, we analysed the effects of environment and aging on astroglial plasticity in the hippocampal dentate gyrus with an optical fractionator.

Acquisition and retrieval of spatial information are hippocampaldependent tasks that can be impaired by structural/functional changes induced by aging (Riedel et al., 1999; D’Hooge \& De Deyn, 2001). Spatial memory decline has been associated with age-related changes in the brain regions involved with spatial learning, e.g. the hippocampal formation (Teather et al., 2002; Frick \& Fernandez, 2003; Rosenzweig \& Barnes, 2003). In the present report, the water maze tests revealed that IA subjects did not exhibit an ability to learn and remember the position of a hidden platform; in contrast, IY, EY and EA individuals did exhibit these abilities, albeit to different extents. We did not find any significant differences in swimming speeds between young and aged mice in either the IC or the EC groups; this suggested that cognitive decline, rather than physical differences, was more likely to explain the differences in performance in the water maze task. Another way to interpret these results could be that the differences in the performances of the young group (EY and IY) in comparison with the aged group (EA and IA) were due to the fact that they started out with different distances traveled (Fig. 2B). However, when a normalized relative scale expressed as an index of 
learning rate (see Materials and methods) is applied to each animal (Fig. 2A), it becomes evident that on average the learning rate is higher in EY and IY in comparison with EA and IA. Indeed, EY and IY presented significant differences in the escape latency at the 3rd day, whereas EA and IA only at the 5th and 7th day, respectively. In agreement, two-way ANOVA confirmed that both environment and aging affected MWM performances.

It has previously been confirmed that episodic-like memory in rats and mice is a hippocampal-dependent task, as it is selectively disrupted by fornix lesions, by lesions of the dorsal and/or ventral hippocampus, by disruption of the CA3 network, or by hippocampal infusion of $N$-methyl-D-aspartate (NMDA) and $\alpha$-amino-3-hydroxy5-methyl-4-isoxazolepropionic acid (AMPA) receptor antagonists (Daumas et al., 2004; Eacott \& Norman, 2004; Ergorul \& Eichenbaum, 2004; Bast et al., 2005; Li \& Chao, 2008). We detected episodic-like memory impairments in both young adult and aged mice raised in IC; thus, we reasoned that the hippocampus could be an important target for the pathophysiological changes associated with IC in female albino Swiss mice. Each hippocampal region contains a distinct population of neurons that express unique molecular profiles (Zhao et al., 2001), and the dentate gyrus seems to be particularly sensitive to age-related changes (Small et al., 2004). Therefore, we speculate that spatial memory deficits in young adult mice may have different pathophysiological mechanisms than those in aged mice, and that both are additive to the influences of an impoverished environment. Indeed, when young adult and aged mice raised in IC were tested for spatial memory and learning with the water maze paradigm, the young adults were able to learn and remember the position of the hidden platform after five training days, but the aged mice were unable to complete the test at this time point. These results suggested that, after 6 months of IC, the hippocampal requirements for spatial learning in young mice could be reactivated with training. As expected, memory capabilities became worse when advanced age was combined with an impoverished environment; under these conditions, learning and memory at the Morris water maze took 7 days to become significant, but all types of episodic-like memories deteriorated. In contrast, aged mice that grew up in enriched environments exhibited relatively unimpaired learning and memory in all memory tests. This suggested that the consolidation and retrieval mechanisms for these memories were spared under enriched environmental conditions.

\section{Dentate gyrus astrocytes, impoverishment, aging and cognitive decline}

The pathophysiological bases for memory impairments have not yet been completely elucidated, but the impact of enriched environment on glial cells has been previously described (Altman \& Das, 1964). In this context the main novelty of the present work is the inclusion of aged mice and the description of how regional gliogenesis changes with aging.

In that light, it is interesting to discuss possible connections between the quantitative astrocytic response and the cognitive protection observed with environmental enrichment in the present report. Our stereological analysis of hippocampal sections from IC aged mice revealed hyperplasia of astrocytes compared with equivalent sections from IC young adults. These differences were associated with a striking cognitive decline in IC aged mice. In contrast, EC aged mice exhibited a higher number of astrocytes in the molecular layer compared with IC aged mice. Interestingly, EA subjects exhibited the ability to form integrated memories in the spatio-temporal context and learned to find and remember the position of the hidden platform in the water maze test. Accordingly, the analysis of the water maze test video revealed that there was a gradient between the EY (who exhibited the best learning rate) and IA (who exhibited no learning) mice. This was evidenced by the intermediate performances of IY and EA individuals. However, the molecular layer of the dentate gyrus was additively affected by both environment and aging; thus, we speculate that astrocytosis induced by environmental enrichment may have a different functional role than that induced by aging. Indeed, it has been demonstrated more recently that astroglia induces neurogenesis from adult neural stem cells (Song et al., 2002), and that astrocytes regulate synapse formation (Mauch et al., 2001; Ullian et al., 2001) and modulation (Haydon, 2001), confirming an active rather than supportive role for astrocytes in adult brains. In addition regional specificity to these cells with distinct subtypes regulating different functions in different regions has been described. For example, Song et al. (2002) found regional differences in astrocytes in neurogenic (dentate gyrus) vs. non-neurogenic (spinal cord) areas. In this context we may interpret that it is possible that different phenotypes of astrocytes in different layers of dentate gyrus may increase selectively as a consequence of enrichment and aging.

In line with this view, the presence of synaptic potentiation via astrocytic glutamate exocytosis at the entorhinal-to-dentate granular cells (perforant pathway) has been described in the dentate molecular layer. By this mechanism astrocytes participate in synaptic tuning in circuits involved in cognitive processing and the control of the mossy fiber-to-CA3 synaptic input (Jourdain et al., 2007). This interpretation would predict more conspicuous changes in the lacunosum molecular layers of CA1 and CA3, target of dense bilateral projections from entorhinal cortex through perforant pathways (van Groen et al., 2003). Indeed, semi-quantitative analysis of astrocyte distribution in CA1 and CA3 of enriched and aging experimental groups (boxed areas of Fig. 5) revealed a much higher expression of GFAP in the stratum lacunosum molecular layer of CA1 and CA3. These data are consistent with the hypothesis that the lacunosum molecular astrocytes may have an important role in synaptic plasticity, and that the experiencedependent GFAP increasing induced by enrichment may be contributing through enhancing this plasticity.

Conversely, even in the absence of neurological disease, a more reactive phenotype of astrocyte is expressed during aging as part of an increased and maintained pro-inflammatory profile that may be associated with cognitive dysfunction; for review, see Godbout \& Johnson (2009). We suggested that IC aged subjects may express higher numbers of pro-inflammatory phenotypes than the aged subjects housed in EC. Morphological 3D reconstructions of astrocytes presently on course in our lab will try to investigate possible morphometric distinctions between enrichment- and aging-induced astrocytes changes.

The notion that aging and impoverished environments may cause similar cellular and molecular alterations in the hippocampus is supported by another study that showed that two nutritional supplements, CDP-choline and uridine monophosphate, prevented spatial memory impairments in both young IC and aged control SpragueDawley rats (Teather \& Wurtman, 2005, 2006). In the present report, we detected differential effects of impoverished environments on the memories of young adult and aged mice. Furthermore, different effects were observed in different layers of the dentate gyrus; in the molecular layer, aging and impoverished environment induced additive astrocytic hyperplasia; in the polymorphic layer, aging induced hyperplasia; and, in the granular layer, neither aging nor environment affected the number of astrocytes. Thus, non-neuronal plasticity was differentially associated with experience in different parts of the laminar circuitry. 
However, because we did not find any simple correlation between the estimations of astrocytes and behavioral performances, quantitative connections between those variables remain open for further investigations. Indeed, the stereological data of the present report are based on five subjects, making it difficult to correlate the behavioral performances with astrocytic changes.

Although a significant increase in the total number of dentate gyrus astrocytes was only observed in young mice raised in EC vs. IC, statistical analysis revealed a tendency of a higher number of astrocytes in IA in comparison to IY subjects (two-way ANOVA, $F=4.24$, $\mathrm{DFn}=1, \mathrm{DFd}=16, P=0.056$ ). This fact is coherent and reproduces previous reports that demonstrated the presence of astrocytosis in the dentate gyrus in aged mice (Mouton et al., 2002; Lei et al., 2003).

A future extension of this study could be to establish, in longitudinal studies, quantitative correlations between pathophysiological changes in astrocytes of other regions of the hippocampus and behavioral performance. This would allow better resolution of the mechanisms of cognitive decline and brain plasticity related to aging.

\section{Non-stereological technical limitations}

Estimations of the number of astrocytes in the dentate gyrus of rats and mice have varied among studies (Nishimura et al., 1995; Pilegaard \& Ladefoged, 1996; Long et al., 1998a; Mouton et al., 2002; Grady et al., 2003; Lei et al., 2003). These contradictions may result from differences in estimation methods, different animal lineages, variations in histological procedures, different stereological protocols, and ambiguities in the definition of the objects and areas of interest. To reduce these possible sources of error when comparing animal groups in the present report, all samples were obtained with the same tissue-processing protocols (perfusion, immunoreaction, dehydration, counterstaining and clearing), and all data were collected and analysed with the same stereological method, software and hardware. To detect possible variations in the criteria for identifying the objects of interest, we underwent checking procedures of the results by having different investigators count the same regions using the same monoclonal GFAP antibody as a selective marker for astrocytes.

As a result, possible variations associated with non-biological sources were reduced to acceptable levels in the present report (Mouton et al., 2002; Slomianka \& West, 2005).

\section{Hormones and astrocytes}

Apart from aging and environment, sexual hormones may change the number of glial cells in the dentate gyrus. In fact, aged C57B16J female mice presented 35\% more astrocytes than age-matched males (Mouton et al., 2002). In the present report, aged females may have been depleted of estrogenic protection; thus, we reasoned that at least part of the astrocytosis might be related to estropause. In support of this reasoning, ovariectomized female mice given an estrogenic replacement showed a reduced number of astrocytes in the dentate gyrus compared with an ovariectomized placebo group (Lei et al., 2003).

Finally, astrocytic plasticity may be affected by corticosteroids, which inhibit astrocytic activation (Liu et al., 2008). In the present report, it could be argued that manipulation-induced stress during the behavioral tests might have altered the plasma corticosteroid levels, and this affected astrocytic plasticity. We did not measure plasma corticosteroid levels; therefore, we cannot exclude the possibility that different levels of corticosteroids might explain the results. However, behavioral tests were applied to all subjects of all experimental groups; thus, we can exclude the possibility that manipulation-induced stress might explain the results.

\section{Conclusion}

The results of the present study demonstrate for the first time within a single report that the effects of environmental enrichment on episodiclike memory are not dependent on age in female albino Swiss mice. Indeed, in the integrative tests, the performances of both young adult and aged mice were improved by enriched environments. Moreover, the water maze test revealed that early onset and long-term environmental enrichment may protect spatial learning and memory from declines induced by aging or environmental impoverishment. A quantitative analysis of the laminar distribution of the astrocytes in the dentate gyrus revealed that the molecular layer developed astrocytosis in response to both environmental enrichment and aging, but the polymorphic layer was altered only by aging. Because aging and enriched environment induced astrocytosis in the molecular layer of the dentate gyrus, it is a temptation to speculate that those two conditions may induce the proliferation of different astrocyte phenotypes. Finally, our work is consistent with previous reports that showed impoverished or enriched environments could prevent (Spangler et al., 1994; Winocur, 1998; van der Staay, 2002) or preserve (Petrosini et al., 2009), respectively, normal cognitive development. Taken together, our findings showed that early onset and extended environmental enrichment, with enhanced sensorimotor, cognitive and social stimulations, induced experience-dependent astrocytic plasticity in the molecular layer of the dentate gyrus. This glial plasticity may represent at least part of the circuitry groundwork for improvements in behavioral performance and maintenance of performance in the aged brain.

\section{Supporting Information}

Additional supporting information may be found in the online version of this article:

Table S1. Optical fractionator counting results for the molecular layer of the dentate gyrus of adult female albino Swiss mice.

Table S2. Optical fractionator counting results for the granular layer of the dentate gyrus of adult female albino Swiss mice.

Table S3. Optical fractionator counting results for the polymorphic layer of the dentate gyrus of adult female albino Swiss mice.

Table S4. Estimated individual unilateral astrocytes numbers $(N)$ with the coefficient of error (CE) for molecular layer of the dentate gyrus of albino Swiss mice.

Table S5. Estimated individual unilateral astrocytes numbers $(N)$ with the coefficient of error (CE) for granular layer of the dentate gyrus of albino Swiss mice.

Table S6. Estimated individual unilateral astrocytes numbers $(N)$ with the coefficient of error (CE) for the polymorphic layer of the dentate gyrus of albino Swiss mice.

Please note: As a service to our authors and readers, this journal provides supporting information supplied by the authors. Such materials are peer-reviewed and may be re-organized for online delivery, but are not copy-edited or typeset by Wiley-Blackwell. Technical support issues arising from supporting information (other than missing files) should be addressed to the authors.

\section{Acknowledgements}

This project was sponsored by Brazilian Government research funds. Grant sponsor: Brazilian Research Council - CNPq; Grant number: 307749/2004-5 and 471077/2007-0 for C.W.P.D. and FINEP, Instituto Brasileiro de Neurociências - IBNnet.

\section{Abbreviations}

EC, enriched conditions; GFAP, glial fibrillary acidic protein; IC, impoverished conditions. 


\section{References}

Altman, J. \& Das, G.D. (1964) Autoradiographic examination of the effects of enriched environment on the rate of glial multiplication in the adult rat brain. Nature, 204, 1161-1163.

Bast, T., da Silva, B.M. \& Morris, R.G. (2005) Distinct contributions of hippocampal NMDA and AMPA receptors to encoding and retrieval of onetrial place memory. J. Neurosci., 25, 5845-5856.

Billard, J.M. \& Rouaud, E. (2007) Deficit of NMDA receptor activation in CA1 hippocampal area of aged rats is rescued by D-cycloserine. Eur. J. Neurosci., 25, 2260-2268

Chadashvili, T. \& Peterson, D.A. (2006) Cytoarchitecture of fibroblast growth factor receptor 2 (FGFR-2) immunoreactivity in astrocytes of neurogenic and non-neurogenic regions of the young adult and aged rat brain. J. Comp. Neurol., 498, 1-15.

Daumas, S., Halley, H. \& Lassalle, J.M. (2004) Disruption of hippocampal CA3 network: effects on episodic-like memory processing in C57BL/6J mice. Eur. J. Neurosci., 20, 597-600.

Dere, E., Huston, J.P. \& De Souza Silva, M.A. (2005a) Episodic-like memory in mice: simultaneous assessment of object, place and temporal order memory. Brain Res. Brain Res. Protoc., 16, 10-19.

Dere, E., Huston, J.P. \& De Souza Silva, M.A. (2005b) Integrated memory for objects, places, and temporal order: evidence for episodic-like memory in mice. Neurobiol. Learn. Mem., 84, 214-221.

Dere, E., Kart-Teke, E., Huston, J.P. \& De Souza Silva, M.A. (2006) The case for episodic memory in animals. Neurosci. Biobehav. Rev., 30, 1206-1224.

D'Hooge, R. \& De Deyn, P.P. (2001) Applications of the Morris water maze in the study of learning and memory. Brain Res. Brain Res. Rev., 36, 60-90.

Dix, S.L. \& Aggleton, J.P. (1999) Extending the spontaneous preference test of recognition: evidence of object-location and object-context recognition. Behav. Brain Res., 99, 191-200.

Duffy, S.N., Craddock, K.J., Abel, T. \& Nguyen, P.V. (2001) Environmental enrichment modifies the PKA-dependence of hippocampal LTP and improves hippocampus-dependent memory. Learn. Mem., 8, 26-34

Eacott, M.J. \& Norman, G. (2004) Integrated memory for object, place, and context in rats: a possible model of episodic-like memory? J. Neurosci., $\mathbf{2 4}$ 1948-1953.

Ergorul, C. \& Eichenbaum, H. (2004) The hippocampus and memory for "what," "where," and "when". Learn. Mem., 11, 397-405.

Frick, K.M. \& Fernandez, S.M. (2003) Enrichment enhances spatial memory and increases synaptophysin levels in aged female mice. Neurobiol. Aging, 24, 615-626.

Godbout, J.P. \& Johnson, R.W. (2009) Age and neuroinflammation: a lifetime of psychoneuroimmune consequences. Immunol. Allergy Clin. North Am., 29, 321-337.

Grady, M.S., Charleston, J.S., Maris, D., Witgen, B.M. \& Lifshitz, J. (2003) Neuronal and glial cell number in the hippocampus after experimental traumatic brain injury: analysis by stereological estimation. J. Neurotrauma, 20, 929-941.

van Groen, T., Miettinen, P. \& Kadish, I. (2003) The entorhinal cortex of the mouse: organization of the projection to the hippocampal formation Hippocampus, 13, 133-149.

Haydon, P.G. (2001) GLIA: listening and talking to the synapse. Nat. Rev., 2, 185-193.

Jourdain, P., Bergersen, L.H., Bhaukaurally, K., Bezzi, P., Santello, M., Domercq, M., Matute, C., Tonello, F., Gundersen, V. \& Volterra, A. (2007) Glutamate exocytosis from astrocytes controls synaptic strength. Nat Neurosci., 10, 331-339.

Junjaud, G., Rouaud, E., Turpin, F., Mothet, J.P. \& Billard, J.M. (2006) Agerelated effects of the neuromodulator D-serine on neurotransmission and synaptic potentiation in the CA1 hippocampal area of the rat. J. Neurochem., 98, 1159-1166

Kempermann, G., Kuhn, H.G. \& Gage, F.H. (1997) More hippocampal neurons in adult mice living in an enriched environment. Nature, 386, 493-495.

Kolb, B. \& Whishaw, I.Q. (1998) Brain plasticity and behavior. Annu. Rev. Psychol, 49, 43-64.

Komitova, M., Perfilieva, E., Mattsson, B., Eriksson, P.S. \& Johansson, B.B. (2002) Effects of cortical ischemia and postischemic environmental enrichment on hippocampal cell genesis and differentiation in the adult rat J. Cereb. Blood Flow Metab., 22, 852-860.

Koopmans, G., Blokland, A., van Nieuwenhuijzen, P. \& Prickaerts, J. (2003) Assessment of spatial learning abilities of mice in a new circular maze Physiol. Behav., 79, 683-693.

Lei, D.L., Long, J.M., Hengemihle, J., O'Neill, J., Manaye, K.F., Ingram, D.K. \& Mouton, P.R. (2003) Effects of estrogen and raloxifene on neuroglia number and morphology in the hippocampus of aged female mice. Neuroscience, 121, 659-666.

Li, J.S. \& Chao, Y.S. (2008) Electrolytic lesions of dorsal CA3 impair episodiclike memory in rats. Neurobiol. Learn. Mem., 89, 192-198.

Liu, W.L., Lee, Y.H., Tsai, S.Y., Hsu, C.Y., Sun, Y.Y., Yang, L.Y., Tsai, S.H. \& Yang, W.C. (2008) Methylprednisolone inhibits the expression of glial fibrillary acidic protein and chondroitin sulfate proteoglycans in reactivated astrocytes. Glia, 56, 1390-1400.

Long, J.M., Kalehua, A.N., Muth, N.J., Calhoun, M.E., Jucker, M., Hengemihle, J.M., Ingram, D.K. \& Mouton, P.R. (1998a) Stereological analysis of astrocyte and microglia in aging mouse hippocampus. Neurobiol. Aging, 19, 497-503.

Long, J.M., Kalehua, A.N., Muth, N.J., Hengemihle, J.M., Jucker, M., Calhoun, M.E., Ingram, D.K. \& Mouton, P.R. (1998b) Stereological estimation of total microglia number in mouse hippocampus. J. Neurosci. Methods, 84, 101-108.

Magistretti, P.J. (2006) Neuron-glia metabolic coupling and plasticity. J. Exp. Biol., 209, 2304-2311.

Markham, J.A. \& Greenough, W.T. (2004) Experience-driven brain plasticity: beyond the synapse. Neuron Glia Biol., 1, 351-363.

Mauch, D.H., Nagler, K., Schumacher, S., Goritz, C., Muller, E.C., Otto, A. \& Pfrieger, F.W. (2001) CNS synaptogenesis promoted by glia-derived cholesterol. Science, 294, 1354-1357.

Morris, R. (1984) Developments of a water-maze procedure for studying spatial learning in the rat. $J$. Neurosci. Methods, 11, 47-60.

Mothet, J.P., Rouaud, E., Sinet, P.M., Potier, B., Jouvenceau, A., Dutar, P., Videau, C., Epelbaum, J. \& Billard, J.M. (2006) A critical role for the glialderived neuromodulator D-serine in the age-related deficits of cellular mechanisms of learning and memory. Aging Cell, 5, 267-274

Mouton, P.R., Long, J.M., Lei, D.L., Howard, V., Jucker, M., Calhoun, M.E. \& Ingram, D.K. (2002) Age and gender effects on microglia and astrocyte numbers in brains of mice. Brain Res., 956, 30-35.

Nishimura, A., Ueda, S., Takeuchi, Y., Sawada, T. \& Kawata, M. (1995) Agerelated decrease of serotonergic fibres and S-100 beta immunoreactivity in the rat dentate gyrus. Neuroreport, 6, 1445-1448.

Perea, G. \& Araque, A. (2007) Astrocytes potentiate transmitter release at single hippocampal synapses. Science, 317, 1083-1086.

Petrosini, L., De Bartolo, P., Foti, F., Gelfo, F., Cutuli, D., Leggio, M.G. \& Mandolesi, L. (2009) On whether the environmental enrichment may provide cognitive and brain reserves. Brain Res. Rev., 61, 221-239.

Pilegaard, K. \& Ladefoged, O. (1996) Total number of astrocytes in the molecular layer of the dentate gyrus of rats at different ages. Anal. Quant. Cytol. Histol., 18, 279-285.

van Praag, H., Kempermann, G. \& Gage, F.H. (2000) Neural consequences of environmental enrichment. Nat. Rev., 1, 191-198.

Prediger, R.D., Franco, J.L., Pandolfo, P., Medeiros, R., Duarte, F.S., Di Giunta, G., Figueiredo, C.P., Farina, M., Calixto, J.B., Takahashi, R.N. \& Dafre, A.L. (2007) Differential susceptibility following beta-amyloid peptide-(1-40) administration in C57BL/6 and Swiss albino mice: evidence for a dissociation between cognitive deficits and the glutathione system response. Behav. Brain Res., 177, 205-213.

Rampon, C. \& Tsien, J.Z. (2000) Genetic analysis of learning behavior-induced structural plasticity. Hippocampus, 10, 605-609.

Rao, S.B., Chetana, M. \& Uma Devi, P. (2005) Centella asiatica treatment during postnatal period enhances learning and memory in mice. Physiol. Behav, 86, 449-457.

Riedel, G., Micheau, J., Lam, A.G., Roloff, E.L., Martin, S.J., Bridge, H., de Hoz, L., Poeschel, B., McCulloch, J. \& Morris, R.G. (1999) Reversible neural inactivation reveals hippocampal participation in several memory processes. Nat. Neurosci., 2, 898-905.

Rosenzweig, E.S. \& Barnes, C.A. (2003) Impact of aging on hippocampal function: plasticity, network dynamics, and cognition. Prog. Neurobiol., 69, $143-179$.

Rosenzweig, M.R. \& Bennett, E.L. (1996) Psychobiology of plasticity: effects of training and experience on brain and behavior. Behav. Brain Res., 78, 57-65.

Shu, S.Y., Ju, G. \& Fan, L.Z. (1988) The glucose oxidase-DAB-nickel method in peroxidase histochemistry of the nervous system. Neurosci. Lett., 85, 169-171.

Sirevaag, A.M. \& Greenough, W.T. (1991) Plasticity of GFAP-immunoreactive astrocyte size and number in visual cortex of rats reared in complex environments. Brain Res., 540, 273-278.

Slomianka, L. \& West, M. (2005) Estimators of the precision of stereological estimates: an example based on the CA1 pyramidal cell layer of rats. Neuroscience, 136, 757-767.

Small, S.A., Chawla, M.K., Buonocore, M., Rapp, P.R. \& Barnes, C.A. (2004) Imaging correlates of brain function in monkeys and rats isolates a 
hippocampal subregion differentially vulnerable to aging. Proc. Natl. Acad. Sci. U S A, 101, 7181-7186.

Soffie, M., Hahn, K., Terao, E. \& Eclancher, F. (1999) Behavioural and glial changes in old rats following environmental enrichment. Behav. Brain. Res., 101, 37-49.

Song, H., Stevens, C.F. \& Gage, F.H. (2002) Astroglia induce neurogenesis from adult neural stem cells. Nature, 417, 39-44.

Spangler, E.L., Waggie, K.S., Hengemihle, J., Roberts, D., Hess, B. \& Ingram, D.K. (1994) Behavioral assessment of aging in male Fischer 344 and brown Norway rat strains and their F1 hybrid. Neurobiol. Aging, 15, 319-328.

van der Staay, F.J. (2002) Assessment of age-associated cognitive deficits in rats: a tricky business. Neurosci. Biobehav. Rev., 26, 753-759.

Teather, L.A. \& Wurtman, R.J. (2005) Dietary CDP-choline supplementation prevents memory impairment caused by impoverished environmental conditions in rats. Learn. Mem., 12, 39-43.

Teather, L.A. \& Wurtman, R.J. (2006) Chronic administration of UMP ameliorates the impairment of hippocampal-dependent memory in impoverished rats. J. Nutr., 136, 2834-2837.

Teather, L.A., Magnusson, J.E., Chow, C.M. \& Wurtman, R.J. (2002) Environmental conditions influence hippocampus-dependent behaviours and brain levels of amyloid precursor protein in rats. Eur. J. Neurosci., 16, 2405-2415.

Todd, K.J., Serrano, A., Lacaille, J.C. \& Robitaille, R. (2006) Glial cells in synaptic plasticity. J. Physiol. Paris, 99, 75-83.

Torres, J.B., Assuncao, J., Farias, J.A., Kahwage, R., Lins, N., Passos, A., Quintairos, A., Trevia, N. \& Diniz, C.W. (2006) NADPH-diaphorase histochemical changes in the hippocampus, cerebellum and striatum are correlated with different modalities of exercise and watermaze performances. Exp. Brain Res., 175, 292-304.

Ullian, E.M., Sapperstein, S.K., Christopherson, K.S. \& Barres, B.A. (2001) Control of synapse number by glia. Science, 291, 657-661.

Viola, G.G., Rodrigues, L., Americo, J.C., Hansel, G., Vargas, R.S., Biasibetti, R., Swarowsky, A., Goncalves, C.A., Xavier, L.L., Achaval, M., Souza, D.O. \& Amaral, O.B. (2009) Morphological changes in hippocampal astrocytes induced by environmental enrichment in mice. Brain Res., 1274, $47-54$

Winocur, G. (1998) Environmental influences on cognitive decline in aged rats. Neurobiol. Aging, 19, 589-597.

Zhao, X., Lein, E.S., He, A., Smith, S.C., Aston, C. \& Gage, F.H. (2001) Transcriptional profiling reveals strict boundaries between hippocampal subregions. J. Comp. Neurol., 441, 187-196. 